Groups Geom. Dyn. 7 (2013), 737-750

DOI $10.4171 / \mathrm{GGD} / 204$
Groups, Geometry, and Dynamics

(C) European Mathematical Society

\title{
On cyclic CAT(0) domains of discontinuity
}

\author{
Eric Swenson*
}

\begin{abstract}
Let $X$ be a CAT(0) space, and $G$ a discrete cyclic group of isometries of $X$. We investigate the domain of discontinuity for the action of $G$ on the boundary $\partial X$.
\end{abstract}

Mathematics Subject Classification (2010). 53C23, 20 F67.

Keywords. CAT(0), Hadamard space, domain of discontinuity.

\section{Introduction}

The idea of a domain of discontinuity was first investigated in the setting of Kleinian groups, discrete groups of isometries of hyperbolic $m$-space, $\mathbb{H}^{m}$. Using the Poincaré ball model of $\mathbb{H}^{m}$, where $\mathbb{H}^{m}$ is the interior of the open unit $m$-ball, we see that the visual boundary of $\mathbb{H}^{m}$ (see Section 2 for definition) is the unit sphere $\mathbb{S}^{m-1}$, and we see that isometries of $\mathbb{H}^{m}$ extend to homeomorphisms of the closed $m$-ball, $\mathbb{H}^{m} \cup \mathbb{S}^{m-1}$.

Let $G$ be a discrete group of isometries of $\mathbb{H}^{m}$, and $\left(g_{i}\right)$ a sequence of distinct elements of $G$. Fixing a point $a \in \mathbb{H}^{m}$, using compactness and passing to a subsequence, we may assume that $g_{i}(a) \rightarrow p \in \mathbb{S}^{m-1}$ and $g_{i}^{-1}(a) \rightarrow n \in \mathbb{S}^{m-1}$. It can be shown that $g_{i}(x) \rightarrow p$ uniformly on compact subsets of $\mathbb{S}^{m-1}-\{n\}$. Since any sequence of elements of $G$ has such a subsequence, we say that $G$ acts as a convergence group on $\mathbb{S}^{m-1}$.

The set of all such $p$ is the limit set of the Kleinian group $G$,

$$
\Lambda G=\left\{p \in \mathbb{S}^{m-1}: \text { there exists }\left(g_{i}\right) \subset G \text { with } g_{i}(x) \rightarrow p \text { for some } x \in \mathbb{H}^{m}\right\}
$$

and the domain of discontinuity of $G, \Omega G=\mathbb{S}^{m-1}-\Lambda G$. Clearly $\Lambda G$ is $G$-invariant and closed, and therefore $\Omega G$ is $G$-invariant and open. Let $p \in \Lambda G$ and $\left(g_{i}\right) \subset G$ with $g_{i}(x) \rightarrow p$ and $g_{i}^{-1}(x) \rightarrow n \in \Lambda G$ for some (any) $x \in \mathbb{H}^{m}$. Then for any $a \in \Omega G, g_{i}(a) \rightarrow p$. Thus if $\Omega G \neq \emptyset$, then $p$ is a limit point of $\Omega G$, and so $\Omega G$ is dense in $\mathbb{S}^{m-1}$.

\footnotetext{
* This work was partially supported by a grant from the Simons Foundation (209403).
} 
We thus arrive at the following result: If $G$ is a discrete group of isometries of hyperbolic $m$-space, $\mathbb{H}^{m}$, then $\partial \mathbb{H}^{m}=\mathbb{S}^{m-1}$ is a disjoint union of the limit set of $G$, $\Lambda G$, and the domain of discontinuity of $G, \Omega G$, where $\Omega G$ is open and either dense or empty. Since $G$ acts as a convergence group on $\mathbb{S}^{m-1}$, the action of $G$ on $\Omega G$ is properly discontinuous (for any compact $C \subset \Omega G,\{g \in G: g(C) \cap C \neq \emptyset\}$ is finite).

This result holds whenever $G$ acts as a discrete convergence group on a compact Hausdorff space, in particular when $G$ is a properly discontinuous group of isometries of a proper $\delta$-hyperbolic space [9].

In this paper we consider the case where the negative curvature ( $\delta$-hyperbolic) condition is relaxed to a non-positive curvature (CAT( 0$)$ ) condition. This result fails in general, as [10] gives an example of a discrete group of isometries of a $\operatorname{CAT}(0)$ space whose limit set contains a nonempty open subset of the boundary, but is not the whole boundary.

The conjecture seems to be that if a cyclic subgroup acts "nicely" on a CAT(0) space and the action on the boundary is not virtually trivial, then there should be an open dense subset of the boundary on which the cyclic subgroup acts properly discontinuously.

The conjecture is realized when the Tits diameter of the boundary is large:

Theorem 4. Let $X$ be a complete CAT(0) space with Tits radius of $\partial X$ more than $3 \pi$ and $|\partial X|>2$. If $h$ is a hyperbolic isometry of $X$, then $\langle h\rangle$ acts properly discontinuously on the open subset $\Omega=\partial X-\left[\bar{B}_{T}\left(h^{+}, \frac{\pi}{2}\right) \cup \bar{B}_{T}\left(h^{-}, \frac{\pi}{2}\right)\right]$. If $\left[\bar{B}_{T}\left(h^{+}, \frac{\pi}{2}\right) \cup \bar{B}_{T}\left(h^{-}, \frac{\pi}{2}\right)\right] \subset \Lambda X$, then $\Omega$ is dense in $\partial X$.

Herein $\bar{B}_{T}(x, r)$ is the closed ball in the Tits metric about $x$ of radius $r$ and $\Lambda X$ is the limits set of the isometry group of $X$.

Theorem 6. Let $X$ be a proper $\mathrm{CAT}(0)$ space with Tits radius more than $3 \pi$, and $h$ a parabolic isometry of $X$. Then there exists $m \in \partial X$, a fixed point of $h$, such that $\langle h\rangle$ acts properly discontinuously on the open dense subset $\Omega=\partial X-\bar{B}_{T}(m, \pi)$ of $\partial X$. If $\bar{B}_{T}(m, \pi) \subset \Lambda X$, then $\Omega$ is dense in $\partial X$.

When the Tits radius is small, we get the following weaker (but much more difficult) result.

Main Theorem. Let $g$ be a hyperbolic isometry of the cocompact proper $\operatorname{CAT}(0)$ space $X$. If $\Omega=\partial X-S_{g} \neq \emptyset$, then $\Omega$ is a dense open subset of $\partial X$, and $\langle g\rangle$ acts discretely on $\Omega$.

Herein $S_{g}$ is the union of $\left\{g^{ \pm}\right\}$and all Tits geodesics of length $\pi$ (if any) between $g^{-}$and $g^{+}$in $\partial X$.

The case where $\partial X=S_{g}$ will not be addressed in this note. 
In the process of proving this main theorem, we obtain the following result which is of independent interest (perhaps more so than our original problem). The version given here is slightly weaker than what we prove, but is easier to state.

Corollary 16. Let $X$ be a proper CAT(0) space with almost extendable geodesics and finite dimensional Tits boundary, and $G$ a group of isometries of $X$. Suppose there is a convex subset $W$ of $X$ satisfying the following conditions:

- $W=Y \times Z$ where $Y \cong \mathbb{E}^{m}$.

- $\partial W$ contains an open subset $U$ of $\partial X$.

- There exists a geodesic ray $R \subset W$ with $R(\infty) \in U$, and a compact $C \subset X$ with $R \subset \bigcup_{g \in G} g(C)$.

Then every point of $\partial Y$ is a spherical suspension point of $\partial X$.

Herein $a$ is a spherical suspension point of $\partial X$ if there exists $b \in \partial X$ and $W \subset \partial X$ such that $\partial X=\{a, b\} * W$, where $*$ is the spherical join. The author wishes to thank M. Kapovich, G. Levitt and K. Ruane for helpful conversations, and the referee and M. Kapovich for suggestions of improvement.

\section{Definitions and basic results}

We refer the reader to [5] or [1] for more details of the following.

Definition. For $X$ a geodesic metric space and $\Delta(a, b, c)$ a geodesic triangle in $X$ with vertices $a, b, c \in X$ there is a comparison triangle $\bar{\Delta}=\Delta(\bar{a}, \bar{b}, \bar{c}) \subset \mathbb{E}^{2}$ with $d(a, b)=d(\bar{a}, \bar{b}), d(a, c)=d(\bar{a}, \bar{c})$ and $d(b, c)=d(\bar{b}, \bar{c})$. We define the comparison angle $\bar{L}_{a}(b, c)=\angle_{\bar{a}}(\bar{b}, \bar{c})$.

Each point $z \in \Delta(a, b, c)$ has a unique comparison point, $\bar{z} \in \bar{\Delta}$. We say that the triangle $\Delta(a, b, c)$ is CAT $(0)$ if for any $y, z \in \Delta(a, b, c)$ with comparison points $\bar{y}, \bar{z} \in \bar{\Delta}, d(y, z) \leq d(\bar{y}, \bar{z})$. The space $X$ is said to be CAT(0) if every geodesic triangle in $X$ is $\operatorname{CAT}(0)$.

If $X$ is CAT(0), notice that for any geodesics $\alpha:[0, r] \rightarrow X$ and $\beta:[0, s] \rightarrow X$ with $\alpha(0)=\beta(0)=a$, the function

$$
\theta(r, s)=\bar{Z}_{r(0)}(\alpha(r), \beta(s))
$$

is an increasing function of $r, s$. Thus $\lim _{r, s \rightarrow 0} \theta(r, s)$ exists and we call this limit $\angle_{a}(\alpha(r), \beta(s))$. It follows that for any $a, b, c \in X$, a CAT(0) space,

$$
\angle_{a}(b, c) \leq \bar{L}_{a}(b, c) .
$$

For the following discussion, we assume that $X$ is a complete CAT(0) space. The (visual) boundary, $\partial X$, is the set of equivalence classes of rays, where rays are 
equivalent if they are within finite Hausdorff distance from each other. Given a ray $R$ and a point $x \in X$, there is a ray $S$ emanating from $x$ with $R \sim S$. Fixing a base point $\mathbf{0} \in X$, we define the visual topology on $\bar{X}=X \cup \partial X$ by taking the basic open sets of $x \in X$ to be the open metric balls about $x$. For $y \in \partial X$, and $R$ a ray from 0 representing $y$, we construct basic open sets $U(R, n, \varepsilon)$ where $n, \varepsilon>0$. We say $z \in U(R, n, \varepsilon)$ if the unit speed geodesic, $S:[0, d(\mathbf{0}, z)] \rightarrow \bar{X}$, from $\mathbf{0}$ to $z$ satisfies $d(R(n), S(n))<\varepsilon$. These sets form a basis for a regular topology on $\bar{X}$ and $\partial X$. For any $x \in X$ and $u, v \in \partial X$, we can define $L_{x}(u, v)$ and $\bar{L}_{x}(u, v)$ by parameterizing the rays $[x, u)$ and $[x, v)$ by $t \in[0, \infty)$ and taking the limit of $\bar{Z}_{x}$ as $t \rightarrow 0$ and $t \rightarrow \infty$ respectively.

For $u, v \in \partial X$, we define $\angle(u, v)=\sup _{p \in X} \angle_{p}(u, v)$. It follows from [5] that $\angle(u, v)=\bar{L}_{p}(u, v)$ for any $p \in X$. Notice that isometries of $X$ preserve the angle between points of $\partial X$. The angle defines a path metric, $d_{T}$ on the set $\partial X$, called the Tits metric, whose topology is finer than the visual topology of $\partial X$. Also $\angle(a, b)$ and $d_{T}(a, b)$ are equal whenever either of them is less than $\pi$. For any $u \in \partial X$, we define $B_{T}(u, \varepsilon)=\left\{v \in \partial X: d_{T}(u, v)<\varepsilon\right\}$ and $\bar{B}_{T}(u, \varepsilon)=\left\{v \in \partial X: d_{T}(u, v) \leq \varepsilon\right\}$.

The set $\partial X$ with the Tits metric is called the Tits boundary of $X$, denoted $T X$. Isometries of $X$ extend to isometries of $T X$

The identity function $T X \rightarrow \partial X$ is continuous, but the identity function $\partial X \rightarrow$ $T X$ is only lower semi-continuous. That is for any sequences $\left(u_{n}\right),\left(v_{n}\right) \subset \partial X$ with $u_{n} \rightarrow u$ and $v_{n} \rightarrow v$ in $\partial X$, then

$$
\underline{\lim } d_{T}\left(u_{n}, v_{n}\right) \geq d_{T}(u, v) .
$$

Lemma 1 ([7], p. 175, Proposition 3.4.3). Let $X$ be a complete CAT(0) space, $v \in \partial X$, and $H \subset X$ a horoball centered at $v$. Then $\bar{H} \cap \partial X=\bar{B}_{T}\left(v, \frac{\pi}{2}\right)$.

Proof. We first show that $\bar{H} \cap \partial X \subseteq \bar{B}_{T}\left(v, \frac{\pi}{2}\right)$. Suppose that $w \in \bar{H} \cap \partial X$ with $\angle(v, w)>\frac{\pi}{2}$. Choose a point $y \in X$ with $\angle y(v, w)>\frac{\pi}{2}$.

Let $R:[0, \infty) \rightarrow X$ be the geodesic ray from $y$ to $v$, and $b_{R}: X \rightarrow \mathbb{R}$ be the Buseman function associated to $R, b_{R}(x)=\lim _{t \rightarrow \infty}[d(x, R(t))-t]$. Note the $b_{R}(y)=0$ and $b_{R}(R(t))=-t$ for $t>0$. By [5], Exercise 8.23(1), p. 272, any horoball based at $v$ is within finite distance of any other horoball based at $v$. It follows that all horoballs based at $v$ will have the same limit points in the boundary. Thus we may assume that $H=b_{R}^{-1}([-1,-\infty))$.

Since $w \in \bar{H}$, there exists $\widehat{w} \in H$ such that $\angle_{y}(v, \widehat{w})>\frac{\pi}{2}$.

We recall [5], Exercise 8.23 (4), p. 273. Choose a geodesic line $\bar{R}: \mathbb{R} \rightarrow \mathbb{E}^{2}$. For each $x \in X$ and $n \in \mathbb{N}$ consider a comparison triangle $\bar{\Delta}(x, R(0), R(n))$ with $\bar{R}(i)$ the comparison point for $R(i)(i=0, n)$ and $x_{n} \in \mathbb{E}^{2}$ the comparison point for $x$. Choose $r_{n} \in \mathbb{R}$ with $\bar{R}\left(r_{n}\right)=\pi_{\bar{R}}\left(x_{n}\right)$ (where $\pi_{\bar{R}}$ is orthogonal projection to the line $\bar{R})$. Then $r_{n} \rightarrow r$ where $b_{R}(x)=-r$. Since $b_{R}(\hat{w}) \leq-1$, for $n \gg 0, r_{n}>0$. It follows that $\angle_{\bar{R}(0)}\left(\bar{R}(n), \hat{w}_{n}\right)<\frac{\pi}{2}$. However $\frac{\pi}{2}<L_{y}(v, \widehat{w})=\angle_{R(0)}(R(n), \hat{w}) \leq$ 
$\bar{Z}_{R(0)}(R(n), \widehat{w})=\angle_{\bar{R}(0)}\left(\bar{R}(n), \widehat{w}_{n}\right)<\frac{\pi}{2}$ which is a contradiction. Thus $\bar{H} \cap \partial X \subseteq$ $\bar{B}_{T}\left(v, \frac{\pi}{2}\right)$.

We now show that $\bar{H} \cap \partial X \supseteq \bar{B}_{T}\left(v, \frac{\pi}{2}\right)$. Since $\bar{H}$ is closed in $\bar{X}$, it suffices to show that $B_{T}\left(v, \frac{\pi}{2}\right) \subset \bar{H}$. Choose $R:[0, \infty) \rightarrow X$ unit speed geodesic ray to $v$, and with associated Buseman function $b_{R}(x)=\lim _{t \rightarrow \infty}[d(x, R(t))-t]$. We may assume that $H=b_{R}^{-1}([0,-\infty))$. Let $y=R(0)$. We note the $b_{R}(y)=0$ and $b_{R}(R(t))=-t$ for $t>0$.

Let $w \in \partial X$ with $\angle(w, v)=\theta<\pi$, and $S:[0, \infty) \rightarrow X$ be the unit speed geodesic from $y$ to $w$. We will show that $S([0, \infty)) \subset H$. Since the function $b_{R}$ is convex and $b_{R} \circ S(0)=0$, it suffices to show that $b_{R} \circ S(s) \leq 0$ for all $s \gg 0$. By [1], Proposition II 4.2, for $s \gg 0 \angle_{S(s)}(y, v)>\frac{\pi}{2}$. Fix such $s \gg 0$ and let $x=S(s)$, so $\angle_{x}(y, v)>\frac{\pi}{2}$.

It suffices to show that $b_{R}(x) \leq 0$. Since $\lim _{t \rightarrow \infty} \angle_{x}(y, R(t))=\angle_{x}(y, v)$, for $t \gg 0, L_{x}(y, R(t))>\frac{\pi}{2}$. Since $\bar{L}_{x}(y, R(t)) \geq L_{x}(y, R(t))$, then for $t \gg 0$, $\bar{Z}_{x}(y, R(t))>\frac{\pi}{2}$ implying that $d(x, R(t))<d(y, R(t))=t$. Thus $[d(x, R(t))-$ $t]<0$ for all $t \gg 0$ and so $b_{R}(x) \leq 0$ as required.

\section{Large Tits radius}

For $A \subset \partial X$, we define

$$
\operatorname{radius}_{T}(A)=\inf \left\{r: A \subset B_{T}(u, r) \text { for some } u \in \partial X\right\} .
$$

If $g$ is an infinite order isometry of $X$, and $\langle g\rangle$ is proper, then $g$ is either hyperbolic or parabolic. When $g$ is hyperbolic, it acts by translation on a line (called an axis of $g$ ) in $X$ with endpoints $g^{+}$(in the direction of translation) and $g^{-}$(see [5], Theorem 6.8, p. 231).

Recall that a hyperbolic isometry $h$ of $X$ is said to be rank 1 if $h$ has an axis $L$ which does not bound a half flat. From [1] we see that $d_{T}\left(h^{+}, \alpha\right)=\infty$ for all $\alpha \neq h^{+}$, so if $X$ has a rank 1 isometry, then $\operatorname{radius}_{T}(\partial X)=\infty$.

Recall a result from [16].

Theorem 2 ([16]). Let $X$ be a complete CAT(0) space and $\left(g_{i}\right)$ a sequence of isometries of $X$ with the property that $g_{i}(x) \rightarrow p \in \partial X$ and $g_{i}^{-1}(x) \rightarrow n \in \partial X$ for any $x \in X$. Then for any $\theta \in[0, \pi]$ and any compact set $K \subset \partial X-\bar{B}_{T}(n, \theta)$, $g_{n}(K) \rightarrow \bar{B}_{T}(p, \pi-\theta)$ (in the sense that for any open $U \supset \bar{B}_{T}(p, \pi-\theta), g_{n}(K) \subset$ $U$ for all $n$ sufficiently large).

This theorem is stated in [16] with stronger hypotheses, but they were not used in the proof. We will refer to this result as $\pi$-convergence. For a given hyperbolic element $h$ with axis $L$, we set $g_{i}=h^{i}, n=L(-\infty)=h^{-}$and $p=L(\infty)=h^{+}$.

The following theorem is due to Ballmann. 
Theorem 3 (Ballmann). If $h$ is a rank 1 isometry of the complete CAT(0) space $X$, then the group $\langle h\rangle$ generated by $h$, acts properly discontinuously on the open set $\partial X-\left\{h^{ \pm}\right\}$which is dense if it is non-empty.

Proof. Let $K$ be a compact subset of $\partial X-\left\{h^{ \pm}\right\}$. The Tits distance from $K$ to $n=h^{-}$ will be infinite. Thus, by $\pi$-convergence, $h^{i}(K) \rightarrow p=h^{+}$and $h^{-i}(K) \rightarrow n=h^{-}$. Thus $\left\{i \in \mathbb{Z}: h^{i}(K) \cap K \neq \emptyset\right\}$ is finite and the action of $\langle h\rangle$ on $\partial X-\left\{h^{ \pm}\right\}$is properly discontinuous. Clearly $\partial X-\left\{h^{ \pm}\right\}$is an open subset and for any $a \in \partial X-\left\{h^{ \pm}\right\}$, $h^{i}(a) \rightarrow h^{+}$and $h^{-i}(a) \rightarrow h^{-}$so $\partial X-\left\{h^{ \pm}\right\}$is dense in $\partial X$.

Definition. For $X$, a complete CAT(0) space, we define the limit set $\Lambda X \subset \partial X$ to be the set $\left\{p \in \partial X\right.$ : there exist $\left(g_{i}\right)$ isometries of $X$ with $g_{i}(x) \rightarrow p$ and $g_{i}^{-1}(x) \rightarrow$ $n$ for some $n \in \partial X$ and for all $x \in X\}$.

The following is a slight generalization of a result of Karlsson [12].

Theorem 4. Let $X$ be a complete $\mathrm{CAT}(0)$ space with $\operatorname{radius}_{T}(\partial X)>3 \pi$ and $|\partial X|>$ 2. If $h$ is a hyperbolic isometry of $X$, then $\langle h\rangle$ acts properly discontinuously on the open subset $\Omega=\partial X-\left[\bar{B}_{T}\left(h^{+}, \frac{\pi}{2}\right) \cup \bar{B}_{T}\left(h^{-}, \frac{\pi}{2}\right)\right]$. If $\left[\bar{B}_{T}\left(h^{+}, \frac{\pi}{2}\right) \cup \bar{B}_{T}\left(h^{-}, \frac{\pi}{2}\right)\right] \subset$ $\Lambda X$, then $\Omega$ is dense in $\partial X$.

Proof. By Theorem 3, we may assume that $h$ is not rank 1. Thus some axis of $h$ bounds a half-flat, which corresponds to a Tits geodesic of length $\pi$ from $h^{+}$to $h^{-}$, so $d_{T}\left(h^{+}, h^{-}\right)=\pi$.

If there is any point of $w \in \partial X$ which is isolated in the Tits metric, $\left(d_{T}(w, v)=\infty\right.$ for all $v \neq w$ ) then, by $\pi$-convergence, the orbit of $w$ under $\langle h\rangle$ is infinite and each element of the orbit will be isolated as well.

By the triangle inequality, for any $q \in \partial X$, there exist $u, v \in \partial X$ such that $d_{T}(q, u), d_{T}(q, v) \geq \pi$ and $d_{T}(u, v)>2 \pi$. If follows from $\pi$-convergence that for any $w \in \Lambda X$ and any neighborhood $W$ of $w$ in $\partial X$, the Tits diameter $\operatorname{diam}_{T}(W)>$ $2 \pi$. Since

$$
\operatorname{diam}_{T}\left[\bar{B}_{T}\left(h^{+}, \frac{\pi}{2}\right) \cup \bar{B}_{T}\left(h^{-}, \frac{\pi}{2}\right)\right] \leq 2 \pi,
$$

$W \not \subset \bar{B}_{T}\left(h^{+}, \frac{\pi}{2}\right) \cup \bar{B}_{T}\left(h^{-}, \frac{\pi}{2}\right)$, so $W \cap \Omega \neq \emptyset$. Thus $\Omega$ is dense in $\Lambda X$. Since closed Tits balls are closed in $\partial X, \Omega$ is open.

For any compact $K \subset \Omega$, by $\pi$-convergence $h^{i}(K) \rightarrow \bar{B}_{T}\left(h^{+}, \frac{\pi}{2}\right)$ and $h^{-i}(K) \rightarrow$ $\bar{B}_{T}\left(h^{-}, \frac{\pi}{2}\right)$. Using this, we can show that $\left\{i \in \mathbb{Z}: h^{i}(K) \cap K \neq \emptyset\right\}$ is finite and so the action of $\langle h\rangle$ on $\Omega$ is properly discontinuous.

Example 5. Notice that $\Omega$ need not be dense in $\partial X$ if we remove the condition that $\bar{B}_{T}\left(h^{+}, \frac{\pi}{2}\right) \cup \bar{B}_{T}\left(h^{-}, \frac{\pi}{2}\right) \subset \Lambda X$. You start with the half plane $\{(x, y): y \geq 0\}$ where $h$ is unit translation in the first coordinate. Now you attach a line at the origin and let $g$ act by translation in that line. You construct the CAT(0) space $X$ by translating this picture by $g$ and $h$. This gives you an action of $\langle g, h\rangle$ on the 
CAT(0) space $X$. The isometry $g$ is a rank 1 hyperbolic element, so $X$ is rank 1 , but $\Omega=\partial X-\left[\bar{B}_{T}\left(h^{+}, \frac{\pi}{2}\right) \cup \bar{B}_{T}\left(h^{-}, \frac{\pi}{2}\right)\right]$ is not dense in the boundary, in fact its closure hits $\left[\bar{B}_{T}\left(h^{+}, \frac{\pi}{2}\right) \cup \bar{B}_{T}\left(h^{-}, \frac{\pi}{2}\right)\right]$ only in $h^{ \pm}$.

Definition. Recall that a metric space is proper if closed metric balls are compact, and a metric space is cocompact if the quotient of the space by the isometry group is compact.

Theorem 6. Let $X$ be a proper CAT(0) space with $\operatorname{radius}_{T}(\partial X)>3 \pi$, and $h$ a parabolic isometry of $X$. There exists $m \in \partial X$ fixed point of $h$ such that $\langle h\rangle$ acts properly discontinuously on the open dense subset $\Omega=\partial X-\bar{B}_{T}(m, \pi)$ of $\partial X$. If $\bar{B}_{T}(m, \pi) \subset \Lambda X$, then $\Omega$ is dense in $\partial X$.

Proof. Clearly $\Omega$ is non-empty and open. By [5], Proposition 8.25, p. 275, there exists $m \in \partial X$ such that $h$ leaves invariant each horoball centered at $m$, which implies that $h$ fixes $m$. Suppose that $\langle h\rangle$ does not act properly discontinuously on $\Omega$. Then there exists $K$, a compact subset of $\Omega$, and a strictly increasing sequence $\left(i_{j}\right) \subset \mathbb{N}$ such that $K \cap h^{i_{j}}(K) \neq \emptyset$ for all $j \in \mathbb{N}$.

Fix $x \in X$. Passing to a subsequence, we may assume that $h^{i_{j}}(x) \rightarrow p \in \partial X$ and $h^{-i_{j}}(x) \rightarrow n \in \partial X$. Since $h$ leaves the horosphere $S$ centered at $m$ passing through $x$ invariant, then $n, p \in \partial S \subset \bar{B}_{T}\left(m, \frac{\pi}{2}\right)$ by Lemma 1 . Since $\partial X$ is normal, there exists $U \subset \partial X$ open with $\bar{B}_{T}(m, \pi) \subset U$ and $U \cap K=\emptyset$. Since $\bar{B}_{T}\left(n, \frac{\pi}{2}\right), \bar{B}_{T}\left(p, \frac{\pi}{2}\right) \subset$ $\bar{B}_{T}(m, \pi) \subset U$, by $\pi$-convergence $h^{i_{j}}(K) \subset U$ for all $j \gg 0$ which implies $h^{i_{j}}(K) \cap K=\emptyset$ for all $j \gg 0$ contradicting the choice of $\left(i_{j}\right)$. Thus $\langle h\rangle$ acts properly on $\Omega$.

By the triangle inequality, for any $q \in \partial X$, there exists $u, v \in \partial X$ such that $d_{T}(q, u), d_{T}(q, v) \geq \pi$ and $d_{T}(u, v)>2 \pi$. If follows from $\pi$-convergence that for any $w \in \Lambda X$ and any neighborhood $W$ of $w$ in $\partial X$, the Tits diameter $\operatorname{diam}_{T}(W)>$ $2 \pi$.

Since $\operatorname{diam}_{T}\left(\bar{B}_{T}(m, \pi)\right) \leq 2 \pi, W \not \subset \bar{B}_{T}(m, \pi)$ so $W \cap \Omega \neq \emptyset$. Thus $\Omega$ is dense in $\Lambda X$.

\section{Small Tits radius}

We now assume that $X$ is a proper $\mathrm{CAT}(0)$ space.

Definition. We say that $a, b \in \partial X$ are antipodes if $d_{T}(a, b) \geq \pi$. The suspension of the antipodes $a$ and $b, S_{a}^{b}$, is the union of $a$ and $b$ together with of all Tits geodesics from $a$ to $b$ of length $\pi$ (if any). Notice that $S_{a}^{b}=S_{b}^{a}$.

Let $a \in \partial X$ and $\left(g_{i}\right)$ a sequence of $X$. We say that $\left(g_{i}\right)$ pulls from $n \in \partial X$ if there is some unit speed geodesic ray $R:[0, \infty) \rightarrow X$ representing $n$, and a sequence 
$\left(s_{i}\right) \subset[0, \infty)$ with $s_{i} \rightarrow \infty$ such that the sequence $\left(g_{i}\left(R\left(s_{i}\right)\right)\right)$ is bounded. Clearly this is independent of the ray chosen. Passing to a subsequence, we may assume that $g_{i}\left(R\left(s_{i}\right)\right) \rightarrow b$, and that the sequence of rays $\left(g_{i}(R)\right)$ (each reparametrized) converges uniformly on compact subsets to a geodesic line $L$ with $g_{i}(-i) \rightarrow L(-\infty)$, and $L(0)=b$. Notice that for some (and so any) $x \in X, g_{i}(x) \rightarrow L(-\infty)$ and $g_{i}^{-1}(x) \rightarrow n$. We say a group of isometries pulls from $n \in \partial X$, if there is a sequence in $G$ which pulls from $n$. Notice that if there is a compact $C \subset X$ such that $R([0, \infty)) \subset \bigcup_{g \in G} g(C)$, then $G$ pulls from $n=R(\infty)$.

For any $x \in \partial X$, passing to a subsequence, we may assume that $g_{i}(x) \rightarrow \hat{x} \in \partial X$. Thus for any compact set $C \subset T X$ we can define $f: C \rightarrow \partial X$ by $f(x)=\hat{x}$. In fact we can define $f$ whenever $C$ has a countable dense subset as a subset of $T X$. By [5], $f: C \rightarrow T X$ is a contraction (Lipschitz with constant one). Notice that $f(n)=L(-\infty)$.

Lemma 7. In the above setting for any $a \in \bar{B}_{T}(n, \pi), f:[n, a] \rightarrow T X$ is an isometric embedding of $[n, a]$ into $S_{L(-\infty)}^{L(\infty)}$, where $[n, a]$ is a Tits geodesic from $n$ to $a$.

Proof. Because $f$ is a contraction, if $d_{T}(n, a)=d_{T}(f(n), f(a))$ then $f:[n, a] \rightarrow$ $T X$ is an isometry. Since $f(n)=L(-\infty)$, it suffices to show that $d_{T}(f(n), f(a))+$ $d_{T}(f(a), L(\infty))=\pi$.

Let $\theta=d_{T}(n, a)$, so by $\pi$-convergence $\pi-\theta \geq d_{T}(f(a), L(\infty))$. Since $f$ is Lipschitz with constant one, $d_{T}(f(n), f(a)) \leq \theta$. However,

$$
\begin{aligned}
\pi=\theta+(\pi-\theta) & \geq d_{T}(f(n), f(a))+d_{T}(f(a), L(\infty)) \\
& \geq d_{T}(L(-\infty), L(\infty)) \geq \pi .
\end{aligned}
$$

Example 8. The function $f$ defined above need not be an embedding on $B_{T}(n, \pi)$. Consider the half flat $Y=\mathbb{R} \times[0, \infty)$. For each $n \in \mathbb{Z}$ glue the quarter flat $X_{n}=[n, \infty) \times[0, \infty)$ by identifying $(a, b) \in Y$ with $(c, d) \in X_{n}$ if $a=c, b=d$, and $\ln (a+1-n) \geq b$. Let $Z$ be the resulting space. $\partial Z$ is broom attached at $\infty$ in the $\mathbb{R}$ factor of $Y$. Notice that $\mathbb{Z}$ acts on $Z\left(1 \in \mathbb{Z}\right.$ sends $X_{n}$ to $\left.X_{n+1}\right)$. Letting $g_{i}$ be subtraction by $i$, we have $f(\partial Z)=\partial Y$.

Definition. Let $g$ be a hyperbolic isometry of the $\operatorname{CAT}(0)$ space $X$. We define $S_{g}=S_{g^{-}}^{g^{+}}$, that is, the suspension of the endpoints of $g$.

Theorem 9. The group $\langle g\rangle$ acts discretely on the open set $\Omega=\partial X-S_{g}$.

Proof. It suffices to show that for any point $a \in \partial X$, the limit points of the $\left\{g^{i}(a)\right.$ : $i \in \mathbb{N}\}$ are in $S_{g}$. Let $b$ be a limit point of $\left\{g^{i}(a): i \in \mathbb{N}\right\}$. Choose an increasing sequence $\left(i_{j}\right) \subset \mathbb{N}$ such that $g^{i_{j}}(a) \rightarrow b$.

Let $L$ be an axis of $g$, then the sequence $\left(g^{i_{j}}\right)$ pulls from $n=L(-\infty)=g^{-}$, leaving $L$ invariant. If $d_{T}(a, n) \geq \pi$, then by $\pi$-convergence $b=L(\infty)=g^{+}$. 
If $d_{T}(a, n)<\pi$, then we construct the function $f:[n, a] \rightarrow T X$ as above and so $f(a)=b$.

By Lemma 7, $f$ embeds $[n, a]$ into $S_{L(-\infty)}^{L(\infty)}=S_{g}$. Thus $b \in S_{g}$.

Definition. A bounded metric space $X$ is almost a product $(K)$ of $Y$ and $Z$ if there is a convex subset $W \cong Y \times Z$ and $X \subset \operatorname{Nbh}(W, K)$.

Definition. For $C>0$, we say that $X$ has almost extendable $(C)$ geodesics if for any $x, y \in X$ there exists $z \in B(y, C)$ such that the geodesic segment $[x, z]$ extends to a ray from $x$. If $X$ has almost extendable geodesics $(C)$ for some $C$, we say $X$ has almost extendable geodesics.

By [11], if $X$ is cocompact then it has almost extendable geodesics.

Lemma 10. Let $X$ be a proper $\mathrm{CAT}(0)$ space $X$ with almost extendable geodesics. Given there is a convex subset $W$ of $X$ where $W=Y \times Z$, and a non-empty open subset $V \subset \partial X$ with $V \subset \partial W$. If the isometries of $X$ pull from a point of $V$, then $X$ is almost a product of $\hat{Y}$ and $\hat{Z}$, convex subsets of $X$. Additionally, if $Y$ is cocompact, then $\hat{Y} \cong Y$.

Proof. Let $C$ be the almost extendability constant. We may assume that $Y$ and $Z$ are (convex) subsets of $W$. Fix a base point $w \in W$. Let $\alpha \in V$ be a point which the isometries of $X$ pulls from. Let $R:[0, \infty) \rightarrow W$ be a geodesic ray with $R(0)=w$ and $R(\infty)=\alpha$. There exist $N \in \mathbb{N}, \varepsilon>0$ such that $U(\alpha, N, \varepsilon) \subset V$.

By definition of pulling from $\alpha$, there is a sequence of isometries $\left(g_{i}\right)$ and $t_{i} \rightarrow$ $\infty$ such that $g_{i}\left(R_{t_{i}}\right) \rightarrow L$ uniformly on compact subset where $R_{t_{i}}$ is the ray $R$ reparametrize to have domain $\left[-t_{i}, \infty\right)$. Using the fact that $X$ is proper and passing to a subsequence, there are unbounded sequences $\left(y_{i}\right) \subset Y,\left(z_{i}\right) \subset Z$ so that $g_{i}\left(w_{i}\right) \rightarrow$ $\widehat{w} \in X$ where $w_{i}=\left(y_{i}, z_{i}\right)$. Using properness and passing to a subsequence, we have that $g_{i}(W) \rightarrow \widehat{W}$, a convex subset of $X$. Let $Y_{i}$ be the copy of $Y$ in $W$ which contains $z_{i}$, and $Z_{i}$ the copy of $Z$ in $W$ which contains $y_{i}$, so that $Y_{i} \cap Z_{i}=\left\{w_{i}\right\}$. Passing to a subsequence, we may assume that $g_{i}\left(Y_{i}\right) \rightarrow \hat{Y}$, a convex subset of $\hat{W}$ and $g_{i}\left(Z_{i}\right) \rightarrow \hat{Z}$, a convex subset of $\hat{W}$. Now let $\hat{a}, \hat{b} \in \hat{W}$. Thus there are sequences $\left(a_{i}\right),\left(b_{i}\right) \subset W$ with $g_{i}\left(a_{i}\right) \rightarrow \hat{a}$ and $g_{i}\left(b_{i}\right) \rightarrow \hat{b}$. For each $i$, let $a_{i}^{Y}=\pi_{Y_{i}}\left(a_{i}\right)$, the projection of $a_{i}$ in $Y_{i}$. Define $b_{i}^{Y}, a_{i}^{Z}$ and $b_{i}^{Z}$ similarly. For each $i, W$ is the metric product of $Y_{i}$ and $Z_{i}$, i.e.,

$$
d\left(a_{i}, b_{i}\right)^{2}=d\left(a_{i}^{Y}, b_{i}^{Y}\right)^{2}+d\left(a_{i}^{Z}, b_{i}^{Z}\right)^{2} .
$$

The sequence $\left(g_{i}\left(a_{i}^{Y}\right)\right)$ will clearly converge to the closest point projection $\pi_{\hat{Y}}(\hat{a})$. Similarly $g_{i}\left(a_{i}^{Z}\right) \rightarrow \pi_{\hat{Z}}(\hat{a}), g_{i}\left(b_{i}^{Y}\right) \rightarrow \pi_{\hat{Y}}(\hat{b})$, and $g_{i}\left(b_{i}^{Z}\right) \rightarrow \pi_{\hat{Z}}(\hat{b})$. It follows that

$$
d(\hat{a}, \hat{b})^{2}=d\left(\pi_{\hat{Y}}(\hat{a}), \pi_{\hat{Y}}(\hat{b})\right)^{2}+d\left(\pi_{\hat{Z}}(\hat{a}), \pi_{\hat{Z}}(\hat{b})\right)^{2},
$$


so $\hat{W}=\hat{Y} \times \hat{Z}$.

When $Y$ admits a cocompact action, we can arrange it so that for some $y \in Y$, and $h_{i}$ isometry of $Y, g_{i}\left(h_{i}(y)\right)$ is bounded and so there is an isometric embedding $h: Y \rightarrow X$ such that $h(Y)=\widehat{Y}$, and similarly for $Z$.

Now we claim that $X \subset \operatorname{Nbh}(\widehat{W}, C)$. Suppose not, then let $x \in X$ with $d(x, \hat{W})>C$. Notice that $g_{i}^{-1}(\bar{B}(x, C)) \rightarrow \alpha$. Thus for $i \gg 0 g_{i}^{-1}(\bar{B}(x, C)) \subset$ $\tilde{U}(\alpha, N, \varepsilon)$.

By almost extendable geodesics $(C)$ there is a point $v_{i} \in g_{i}^{-1}(\bar{B}(x, C))$ such that $v_{i}$ is on a ray $S:[0, \infty) \rightarrow X$ from our base point $w$. Notice that for $i \gg 0$, $d\left(x, g_{i}(W)\right)>C$, so in that case $d\left(v_{i}, W\right)>0$, so $S([0, \infty)) \not \subset W$.

Since the (unit speed geodesic) rays based at $w$ give unique representatives of $\partial X$ and every element of $\partial W$ is represented by a ray in $W$ from $w$, this implies that $S(\infty) \notin \partial W$, but $S(\infty) \in U(\alpha, N, \varepsilon) \subset \partial W$, which is a contradiction.

Corollary 11. Let $X$ be a proper CAT(0) space with almost extendable geodesics and $W$ a convex subset with cocompact stabilizer in the isometry group of $X$. If some nonempty open subset of $\partial X$ is in $\partial W$, then $X$ lies in a uniform neighborhood of $W$, so $\partial X=\partial W$.

Proof. In the proof of Lemma 10, we may choose the sequence $\left(g_{i}\right)$ in the stabilizer of $W$, so $\widehat{W}=W$.

Unfortunately, the previous result raises more questions than it answers

Question. If $X$ and $Y$ are cocompact proper CAT(0) spaces and $U \subset \partial X, V \subset \partial Y$ non-empty open subsets with $U \cong V$, is $\partial X \cong \partial Y$ ? (Here $\cong$ is a homeomorphism in cone topology and an isometry in the Tits metric.)

What if additionally we have a group $G$ acting geometrically on both $X$ and $Y$ ? The answer is clearly yes if $X$ or $Y$ is rank 1 and the isomorphism $U \cong V$ is $G$-equivariant.

See [6] and [15] for related questions.

We now generalize part of a result of Lytchak to our setting.

Theorem 12. [14] Let $Z$ be a geodesically complete finite dimensional CAT(1) space. Then $Z$ has a unique decomposition $Z=\mathbb{S}^{n} * G_{1} * \cdots * G_{k} * Y_{1} * \cdots * Y_{m}$ where $G_{j}$ is a thick irreducible building and $Y_{j}$ is an irreducible (via spherical join) non-building.

In our setting, the Tits boundary $T X$ is not geodesically complete. Nonetheless, we obtain the following result: If $Z$ is a finite dimensional CAT(1) space then there is a unique decomposition $Z=S^{n} * Y$ where $Y$ doesn't have a sphere as a spherical join factor.

Lemma 13. If $Y$ and $Z$ are $\mathrm{CAT}(0)$ spaces and $F$ is a flat sector in $Y \times Z$, then $\pi_{Y}(F)$ is a flat sector in $A$. 
Proof. Since a flat sector is the nested union of flat triangles (and this is sufficient), it suffices to show that the projection of a flat triangle is a flat triangle.

Let $a b c$ be a triangle in a $\mathrm{CAT}(0)$ space. Consider the following condition: For $t \in(0,1)$ if $e \in[a, b]$ and $f \in[a, c]$ with $d(a, e)=t d(a, b)$ and $d(a, f)=t d(a, c)$ then $d(e, f)=t d(b, c)$. Notice that if $a b c$ is flat, then this condition is satisfied by similar triangles. If on the other hand this condition is satisfied, then by similar triangles, the Euclidian comparison angle $\bar{L}_{a}(b, c)=\bar{Z}_{a}(e, f)$. It follows that $\angle_{a}(b, c)=\bar{\angle}_{a}(b, c)$ and so the triangle $a b c$ is flat.

Thus we may assume that $a b c$ is a triangle in satisfying this condition. For $t \in(0,1)$ choose $e$ and $f$ as above. Let $a_{1}, b_{1}, c_{1}, e_{1}, f_{1}$ be the projections of $a$, $b, c, e, f$ respectively into $Y$ and $a_{2}, b_{2}, c_{2}, e_{2}, f_{2}$ the projections of $a, b, c, e$, $f$ respectively into $Z$. By similar Euclidean triangles $d\left(a_{i}, e_{i}\right)=t d\left(a_{i}, b_{i}\right)$ and $d\left(a_{i}, f_{i}\right)=t d\left(a_{i}, c_{i}\right)$ for $i=1,2$.

By hypothesis $d(e, f)=t d(b, c)$, so $d(e, f)^{2}=t^{2} d(b, c)^{2}$. Thus,

$$
d\left(e_{1}, f_{1}\right)^{2}+d\left(e_{2}, f_{2}\right)^{2}=t^{2} d\left(b_{1}, c_{1}\right)^{2}+t^{2} d\left(b_{2}, c_{2}\right)^{2} .
$$

By the CAT(0) inequality applied to the triangles $a_{i} b_{i} c_{i}, i=1,2, d\left(e_{i}, f_{i}\right) \leq$ $t d\left(b_{i}, c_{i}\right)$ for $i=1,2$. It follows that $d\left(e_{i}, f_{i}\right)=t d\left(b_{i}, c_{i}\right)$ for $i=1,2$, and so the triangles $a_{i} b_{i} c_{i}$ are flat for $i=1,2$.

Definition ([5], Corollary 5.11, p. 62). For $Y$ and $Z$, metric spaces of diameter $\leq \pi$, we define $Y * Z$ to be the quotient of the space $Y \times Z \times\left[0, \frac{\pi}{2}\right]$ by the identifications $(y, z, 0)=(y, \hat{z}, 0)$ and $\left(y, z, \frac{\pi}{2}\right)=\left(\hat{y}, z, \frac{\pi}{2}\right)$. We denote the class $(y, z, \theta)$ by $y \cos \theta+z \sin \theta$, so $(y, z, 0)=y$ and $\left(y, z, \frac{\pi}{2}\right)=z$, and so we have $Y, Z \subset Y * Z$. For $u=y \cos \theta+z \sin \theta, u^{\prime}=y^{\prime} \cos \theta^{\prime}+z^{\prime} \sin \theta^{\prime} \in Y * Z$ we define $d\left(u, u^{\prime}\right)$ by

$$
\cos \left(d\left(u, u^{\prime}\right)\right)=\cos \theta \cos \theta^{\prime} \cos \left(d\left(y, y^{\prime}\right)\right)+\sin \theta \sin \theta^{\prime} \cos \left(d\left(z, z^{\prime}\right)\right) .
$$

Clearly for $\theta \neq 0, \frac{\pi}{2}, d\left(u, u^{\prime}\right)=\pi$ if and only if $d\left(y, y^{\prime}\right)=\pi=d\left(z, z^{\prime}\right)$ and $\theta+\theta^{\prime}=\frac{\pi}{2}$.

Lemma 14. Let $\alpha$ be a geodesic in $A * B$ where $A$ and $B$ are $\mathrm{CAT}(1)$ spaces. Notice that $A$ and $B$ are $\pi$-convex subsets of $A * B$. If the image of $\alpha$ misses $B$, then the projection of $\alpha$ to $A$ is a geodesic.

Proof. Let $Y=C_{0}(A)$ and $Z=C_{0}(B)$ be the Euclidian cones on $A$ and $B$ respectively. By [5], $C_{0}(A * B)=X \cong Y \times Z$ and so $A * B=\partial(Y \times Z)$. By [5], Proposition 5.15, p. 64, it suffices to show that the projection of $\alpha$ to $A$ is a local Tits geodesic. There is a flat sector $F \subset X$ based at the cone point 0 of $X$ with $\partial F=\alpha$. We can think of $Y$ and $Z$ as being convex subsets of $X$. By Lemma 13, the projection $F_{Y}$ of $F$ into $Y$ is a Euclidean sector based at the cone point of $Y$ (which is 0 of course). By the proof of [5], Proposition 5.15, p. 64, the $\partial F_{Y}$ will be the projection of $\alpha$ to $A$. It follows that this projection is a Tits geodesic in $A$. 
The following can also be done using the main result of [8].

Theorem 15. If $Z$ is a finite dimensional complete CAT(1) space, then there is a unique decomposition $Z=S * D$, where $S \cong \mathbb{S}^{n}$ and $D$ does not have a non-empty sphere as a spherical join factor.

Proof. Suppose that $Z=\mathbb{S}^{k} * U$ and $Z=\{a, b\} * A$, where $\{a, b\} \not \subset \mathbb{S}^{k}$. It follows that $a$ is the unique antipode to $b$ in $Z$ and visa versa (so $\{a, b\} \cap \mathbb{S}^{k}=\emptyset$ ). By the metric on $Z=\mathbb{S}^{k} * U$, we have $a_{1}, b_{1} \in \mathbb{S}^{k}, a_{2}, b_{2} \in U$ and $\theta \in(0, \pi / 2]$ with $a=a_{1} \cos \theta+a_{2} \sin \theta$ and $b=b_{1} \sin \theta+b_{2} \cos \theta$. If follows that $a_{2}$ is the unique antipode of $b_{2}$ in $U$ and visa versa (and similarly for $a_{1}$ and $b_{1}$ in $\mathbb{S}^{k}$ ).

Since $Z=\{a, b\} * A$, it follows that $Z=S_{a}^{b}$, and every point of $Z$ is on a Tits geodesic from $a$ to $b$. Fix $u \in U \subset \mathbb{S}^{k} * U$; the point $u$ is on a Tits geodesic from $a$ to $b$ which misses $\mathbb{S}^{k}$. By Lemma 13, the projection of this Tits geodesic into $U$ is a Tits geodesic from $a_{2}$ to $b_{2}$ passing through $u$. It follows that $U$ is the union of all Tits geodesics in $U$ from $a_{2}$ to $b_{2}$. This implies by [14], 4.1, that $U=\left\{a_{2}, b_{2}\right\} * W$, where $W$ is the set of all points of $U$ at Tits distance $\pi / 2$ from both $a_{2}$ and $b_{2}$. Thus by [5], Proposition 5.15, p. 64, $Z=\mathbb{S}^{k} *\left(\left\{a_{2}, b_{2}\right\} * W\right)=\left(\mathbb{S}^{k} *\left\{a_{2}, b_{2}\right\}\right) * W=$ $\mathbb{S}^{k+1} * W$. Since $Z$ is finite dimensional, this process must terminate, and so for some $n, Z=\mathbb{S}^{n} * Y$, where $Y$ does not have a non-empty sphere as a spherical join factor. Notice by our construction that

$$
S=\{a \in Z: Z \text { is a spherical suspension with suspension point } a\} .
$$

Thus this decomposition is canonical.

Corollary 16. Suppose that the Tits boundary, $T X$, is finite dimensional. Under the hypothesis of Lemma 10 , if $Y \cong \mathbb{E}^{m}$ for some $m$, then $\partial Y \subset S$, the set of suspension points of $\partial X$.

Proof. By $\partial X=S * D$ as in Theorem 15. Since $\mathbb{E}^{n}$ admits a cocompact isometric group action, $\hat{Y} \cong Y \cong \mathbb{E}^{n} . \partial X=\partial(\hat{Y} \times \widehat{Z})=(\partial Y) *(\partial \widehat{Z})$. Since $\partial \hat{Y} \cong$ $\partial \mathbb{E}^{n}=S^{n-1}$, we have $\partial \hat{Y} \subset S$. Let $\alpha \in \partial Y-S$, so $d_{T}(\alpha, D)<\pi / 2$. We may assume $g_{i}(\alpha) \rightarrow \beta$ preforce with $\beta \in \partial \hat{Y} \subset S$. However $g_{i}(D)=D$ for all $i$, so $d_{T}(\beta, D) \leq d_{T}(\alpha, D)<\pi / 2$, but that is a contradiction since every point of $S$ has distance $\pi / 2$ from every point of $D$. Thus $\partial Y \subset S$.

Definition. We say a group $G$ acts discretely on a space $X$ if every orbit is a discrete subset. This is strictly weaker than saying that a group acts properly discontinuously.

Theorem 17. Let $g$ be a hyperbolic isometry of the proper $\mathrm{CAT}(0)$ space $X$. Suppose that $T X$ is finite dimensional and the isometry group of $X$ pulls from a dense subset of $S_{g}$. If $\Omega=\partial X-S_{g} \neq \emptyset$, then $\Omega$ is a dense open subset of $\partial X$, and $\langle g\rangle$ acts discretely on $\Omega$. 
Proof. By Theorem 9, $\langle g\rangle$ acts discretely on the open set $\Omega$. We must show that $\Omega$ is dense whenever it is non-empty.

Suppose that $\Omega$ is not dense. Then there is an open subset $V$ of $\partial X$ with $V \subset \partial S_{g}$. Recall that $S_{g} \cong \mathbb{R} \times Z$ for some $Z$, where the endpoints of $\mathbb{R}$ are $g^{ \pm}$. Thus by Corollary $16, g^{ \pm} \in S$, the set of suspension points of $\partial X$. It follows that $S_{g}=\partial X$ as required.

Main Theorem. Let $g$ be a hyperbolic isometry of the cocompact proper CAT(0) space $X$. If $\Omega=\partial X-S_{g} \neq \emptyset$, then $\Omega$ is a dense open subset of $\partial X$, and $\langle g\rangle$ acts discretely on $\Omega$.

Proof. By [13], $T X$ is finite dimensional. Since the isometry group of $X$ is cocompact, it pulls from every point of $\partial X$, and Theorem 17 applies.

\section{References}

[1] W. Ballmann, Lectures on spaces of nonpositive curvature. DMV Seminar 25, Birkhäuser Verlag, Basel 1995. Zbl 0834.53003 MR 1377265

[2] W. Ballmann and M. Brin, Orbihedra of nonpositive curvature. Inst. Hautes Études Sci. Publ. Math. 82 (1995), 169-209. Zbl 0866.53029 MR 1383216

[3] W. Ballmann and S. Buyalo, Periodic rank one geodesics in Hadamard spaces. In Geometric and probabilistic structures in dynamics, Contemp. Math. 469, Amer. Math. Soc., Providence, RI, 2008, 19-27. Zbl 1167.53034 MR 2478464

[4] M. Bestvina, Local homology properties of boundaries of groups. Michigan Math. J. 43 (1996), 123-139. Zbl 0872.57005 MR 1381603

[5] M. R. Bridson and A. Haefliger, Metric spaces of non-positive curvature. Grundlehren Math. Wiss. 319, Springer-Verlag, Berlin 1999. Zbl 0988.53001 MR 1744486

[6] C. B. Croke and B. Kleiner, Spaces with nonpositive curvature and their ideal boundaries. Topology 39 (2000), 549-556. Zbl 0959.53014 MR 1746908

[7] P. B. Eberlein, Geometry of nonpositively curved manifolds. Chicago Lectures in Math., University of Chicago Press, Chicago, IL, 1996. Zbl 0883.53003 MR 1441541

[8] T. Foertsch and A. Lytchak, The de Rham decomposition theorem for metric spaces. Geom. Funct. Anal. 18 (2008), 120-143. Zbl 1159.53026 MR 2399098

[9] E. M. Freden, Negatively curved groups have the convergence property I. Ann. Acad. Sci. Fenn. Ser. A I Math. 20 (1995), 333-348. Zbl 0847.20031 MR 1346817

[10] S. Geninska, Examples of infinite covolume subgroups of $\operatorname{PSL}(2, \mathbb{R})^{r}$ with big limit sets. Math. Z. 272 (2012), 389-404. Zbl 1252.11040 MR 2968231

[11] R. Geoghegan and P. Ontaneda, Boundaries of cocompact proper CAT(0) spaces. Topology 46 (2007), 129-137. Zbl 1124.20026 MR 2313068

[12] A. Karlsson, On the dynamics of isometries. Geom. Topol. 9 (2005), 2359-2394. Zbl 1120.53026 MR 2209375 
[13] B. Kleiner, The local structure of length spaces with curvature bounded above. Math. Z. 231 (1999), 409-456. Zbl 0940.53024 MR 1704987

[14] A. Lytchak, Rigidity of spherical buildings and joins. Geom. Funct. Anal. 15 (2005), 720-752. Zbl 1083.53044 MR 2221148

[15] C. P. Mooney, Examples of non-rigid CAT(0) groups from the category of knot groups. Algebr. Geom. Topol. 8 (2008), 1666-1689. Zbl 1163.20028 MR 2448867

[16] P. Papasoglu and E. Swenson, Boundaries and JSJ decompositions of CAT(0)-groups. Geom. Funct. Anal. 19 (2009), 558-590. Zbl 1226.20038 MR 2545250

[17] K. E. Ruane, Dynamics of the action of a CAT(0) group on the boundary. Geom. Dedicata 84 (2001), 81-99. Zbl 0984.20027 MR 1825346

[18] E. L. Swenson, A cut point theorem for CAT(0) groups. J. Differential Geom. 53 (1999), 327-358. Zbl 1038.20029 MR 1802725

Received June 1, 2010; revised April 16, 2012

E. Swenson, Department of Mathematics, Brigham Young University, Provo, UT 84602, U.S.A.

E-mail: eric@math.byu.edu 